\title{
Admittance Dependence of the Infinite Cylindrical Antenna Upon Exciting Gap Thickness ${ }^{1}$
}

\author{
Edmund K. Miller \\ High Altitude Engineering Laboratory, Department of Aerospace Engineering, \\ University of Michigan, Ann Arbor, Mich. 48105, U.S.A.
}

(Received January 9, 1967; revised May 8, 1967)

\begin{abstract}
The admittance of an infinite cylindrical antenna is calculated as a function of exciting frequency and exciting gap thickness. The results obtained show that an asymptotic analytical expression derived for the susceptance by Chen and Keller and also later by Fante, is strictly valid only in the limit of vanishing gap thickness. An altenate analytical form is presented for the susceptance, which removes this restriction and which gives good agreement with the correct susceptance values obtained in this study.
\end{abstract}

\section{Introduction}

The admittance of a cylindrical antenna of finite or infinite length, fed by a circumferential voltage gap, is a subject which has received a great deal of attention. A solution for the antenna current can be exactly obtained only for the infinite antenna, the finite antenna current being determined by an integral equation. The reason for this is the reflected current waves, which are set up by the ends of the finite antenna, but which are absent from the infinite antenna.

While the current, and thus the admittance, of the infinite and finite antennas may be very different, they do have in common the feedpoint singularity associated with an infinitesimal (delta function) exciting gap in the outward traveling current wave. As a result, the change in antenna susceptance that results from changing the gap thickness might be expected to be similar for both antennas. Whether or not the dependence of the susceptance upon gap thickness is significant will depend upon the relative importance of other determining parameters such as antenna radius, and in addition for the finite antenna, the antenna length. The purpose of the work, which is discussed here, is to investigate, using a numerical approach, the admittance variation of the infinite antenna as a function of the gap thickness, as well as the excitation frequency.

It will be demonstrated from some numerical results to be presented that a condition for the asymptotic form derived for the antenna susceptance by Chen and Keller (1962) and also subsequently, by Fante (1966a), to be valid is that $\delta \rightarrow 0$ rather than $k \delta \ll 1$, where $k$ is the wave number and $\delta$ the gap thickness. In addition, the numerical analysis here is not restricted to finding an average gap current in the

\footnotetext{
'The research reported in this paper was supported by the National Aeronautics and Space Administration under Headquarters contract No. NASr $-54(05)$.
}

vicinity of an infinitesimal gap, from which to estimate the susceptance, as was done by Duncan (1962), but. the explicit effect of varying the gap thickness can be obtained. Values are also readily obtained for the antenna conductance, for which some numerical results are presented. The approach taken here is simply a straightforward numerical evaluation of the equation for the antenna current, without any approximations to the integrand as has been done in the papers quoted.

\section{Formulation}

The current on the surface of an infinite antenna of radius $a$, whose axis is coincident with the $z$ axis of a cylindrical $(\rho, \varphi, z)$ coordinate system, and which is excited by a voltage $V_{0} e^{i \omega t}$, may be expressed by (Duncan, 1962),

$$
I(z)=-2 i a \omega \epsilon \int_{0}^{\infty} \cos (\beta z) \frac{S(\beta)}{\lambda} \frac{H_{l}^{(2)}(\lambda a) d \beta}{H_{0}^{(2)}(\lambda a)}
$$

with $\epsilon$ the permittivity of the medium surrounding the antenna, $H_{n}^{(2)}$ the Hankel function of second kind and order $n$, and

$$
\begin{aligned}
\lambda^{2} & =k^{2}-\beta^{2} \\
k & =\frac{\omega}{c}=\omega \sqrt{\mu \epsilon}=2 \pi f \sqrt{\mu \epsilon} \\
S(\beta) & =V_{0} \frac{\sin (\beta \delta / 2)}{(\beta \delta / 2)}
\end{aligned}
$$

where $\delta$ is the gap thickness. The medium permeability is given by $\mu$, and $c$ is the propagation velocity of light in the medium which we take to be free space. 
The real part of (1), which for $V_{0}=1$ and $z=\delta / 2$ is the conductance, is given by the finite integration range 0 to $k$, since for $\beta>k$, the ratio $H_{1}^{(2)} / H_{0}^{(2)}$ becomes pure imaginary, cancelling out the $i$ arising from $\lambda$, so that the integrand function is pure real. The conductance is consequently more easily found than the susceptance, requiring a finite range of integration, and encountering only an integrable singularity at $\beta=k$ (see Duncan, 1962). In addition, since $k \delta \ll 1$ is required for the assumption of a uniform electric field across the gap to be valid, we see that $S(\beta)$ will be equal to unity over the range of integration determining the conductance, which is thus independent of gap width.

Calculating the susceptance from (1) is not so straightforward, however, because nonintegrable singularities of opposite sign are found near $\beta=k$ in the imaginary portion of the integral, and, an important consideration in a numerical treatment, the range of integration is much greater. If, in particular, $\delta=0$, then when $\beta \gg k$ and $\beta a \gg 1, H_{1}^{(2)} / H_{0}^{(2)} \lambda \rightarrow-\beta^{-1}$, and the current at the source is infinite, since $\int^{x} d \beta / \beta$ is not convergent. For nonzero $\delta$, the current is no longer singular, but the range of integration is still very large, on the order of $(k \delta)^{-1}$ times that required to find the conductance.

The approach taken here to numerically evaluate (1) without any restrictions other than assuming a nonzero $\delta$, is to integrate upwards along the imaginary $\beta$ axis to about $\beta=k$, and then integrate parallel to the real $\beta$ axis until the desired convergence has occurred. It is not necessary to return the integration to the real axis, since the contribution to the integral of the neglected segment is negligible at the large value of $\beta$ where the integration is terminated. The integral is considered to have converged when the normalized truncation error that arises from terminating the integration at a finite $\beta$ value, is less than some acceptable normalized error. The integration was carried out using the Romberg integration scheme discussed by Ralston (1965, p. 121). A computer routine that automatically chose the interval size so that the desired convergence took place within each interval with no more than 5 abscissa points, was developed. This scheme was consistently found to select the smallest interval sizes at points where the integrand function is nearly singular, with a resulting maximum to minimum abscissa-spacing ratio as high as $10^{6}$ or $10^{i}$. In the next section we present some numerical results for the admittance calculations.

\section{Numerical Results}

In figure $I$ is shown the susceptance, $B$, of an antenna of $1 . \mathrm{cm}$ radius operated at $1 \mathrm{MHz}(K=k a$ $=2.0958 \times 10^{-4}$ ) as a function of the gap thickness which varies from $0.1 \mathrm{~cm}$ to $10^{-4} \mathrm{~cm}$. For greater numerical accuracy, the susceptance of the antenna, as well as the conductance, $G$, is given in table 1 .
We may observe from figure 1 that the susceptance, in the range $\delta=10^{-1}$ to $10^{-4}$, varies logarithmically, and may be represented as

$$
B=B_{0}+\Delta B \ln (\delta),
$$

where $B_{0}$ and $\Delta B$ may be functions of $K$. With $\delta$ expressed in $\mathrm{cm}$, we may solve for $B_{0}$ and $\Delta B$ from the data in table 1 to get

$$
\begin{aligned}
B_{0} & =2.1612 \times 10^{-4} \text { mhos, } \\
\Delta B & =-1.1111 \times 10^{-6} \text { mhos, }
\end{aligned}
$$

where $K=2.0958 \times 10^{-4}$.

Chen and Keller, and Fante, derived an approximate closed-form expression for the susceptance, which is given by

TABLE 1

\begin{tabular}{c|c|c}
\hline \multicolumn{3}{|c}{$K=2.0958 \times 10^{-4}$} \\
\hline$\delta(\mathrm{cm})$ & $C \times 10^{-4}$ & $B \times 10^{3}$ \\
\hline $10^{-1}$ & 1.0309 & 0.21870 \\
$10^{-2}$ & 1.0309 & 0.22124 \\
$10^{-3}$ & 1.0309 & 0.22379 \\
$10^{-4}$ & 1.0309 & 0.22635 \\
\hline
\end{tabular}

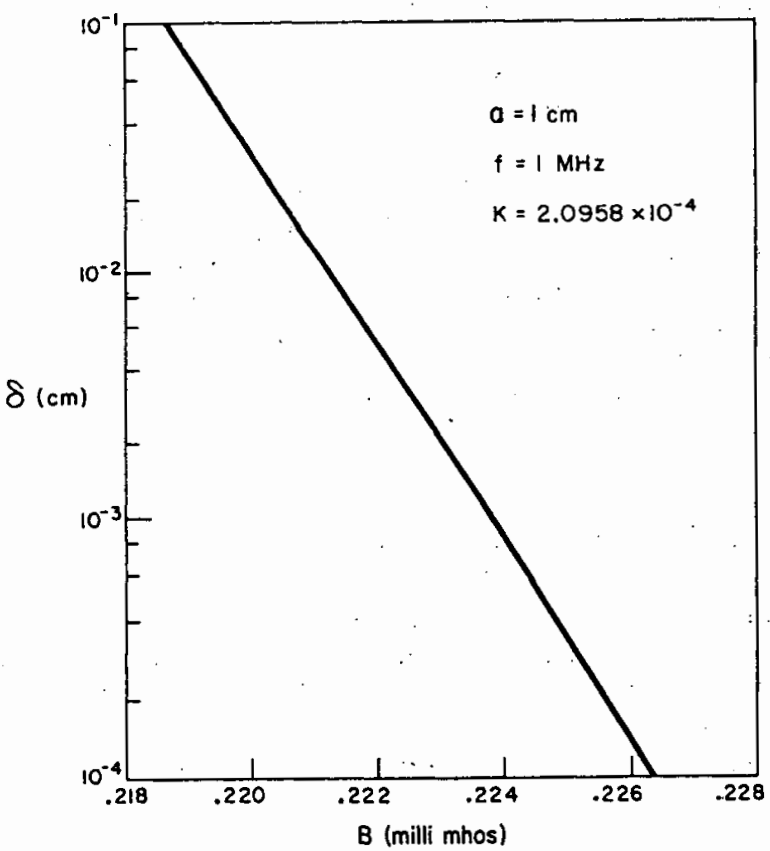

Figure 1. Variation of susceptance B as a function of exciting gap thickness $\delta$, at $1 M H z,\left(\mathrm{~K}=2.0958 \times 10^{-1}\right)$ with an antenna radius a of $1 \mathrm{~cm}$. 


$$
\widetilde{B}=-2 a \omega \epsilon \ln (\mathrm{k} \delta / 2)
$$

where we denote the susceptance by $\widetilde{B}$ to differentiate it from the purely numerical results which are obtained in our approach. We see that (4) may be put in the form of (2), so that

$$
\begin{gathered}
\tilde{B}_{0}=-2 a \omega \epsilon \ln (k / 2), \\
\Delta \tilde{B}=-2 a \omega \epsilon .
\end{gathered}
$$

Solving for $\widetilde{B}_{0}$ and $\Delta \widetilde{B}$ from (5) we get

$$
\begin{aligned}
& \widetilde{B}_{0}=1.0106 \times 10^{-5} \text { mhos } \\
& \tilde{\Delta} \mathrm{B}=-1.1126 \times 10^{-6} \text { mhos }
\end{aligned}
$$

again with $K=2.0958 \times 10^{-4}$. While the $\Delta B$ values $(3 \mathrm{~b})$ and $(6 \mathrm{~b})$ are in quite good agreement, the $\widetilde{B}_{0}$ values are very different and it would appear that a term not containing $\delta$ should be included in (4).

If we examine Fante's derivation more carefully, we find that in (23) of his paper, he neglects a term given by (Fante also neglects the $\Gamma$ in this term, but subsequently corrected this omission; Fante, 1966b)

$$
-0.9\left[K \ln \left(\Gamma^{2} K^{2} / 2\right)\right]^{-1} \cdot 2 a \omega \epsilon
$$

with $\ln \Gamma=$ Euler's constant $(0.577216)$, on the basis that this term is small compared with $\ln (k \delta)$. It is obvious, however, with $K \sim 10^{-4}$ and $k \delta \sim 10^{-6}$ that the neglected term is larger than the one retained. If we keep the term in question, then we obtain from Fante's approach

$$
\tilde{B}=-2 a \omega \epsilon\left[0.9[2 K \ln (\Gamma K / \sqrt{2})]^{-1}+\ln (k / 2)+\ln (\delta)\right],
$$

so that

$$
\widetilde{B}_{0}=-2 a \omega \epsilon\left[0.9[2 K \ln (\Gamma K / \sqrt{2})]^{-1}+\ln (k / 2)\right] .
$$

with $\Delta \tilde{B}$ given by $(5 \mathrm{~b})$. We now find $\tilde{B}_{0}$, for $K$ $=2.0958 \times 10^{-4}$, to be

$$
\widetilde{B}_{0}=3.0012 \times 10^{-4} \mathrm{mhos},
$$

which is now more nearly in agreement with (3a) than was (6a). It is apparent that for sufficiently small values of $\delta$, the susceptance will be dominated by the $\ln (k \delta)$ term, and (4) is then approximately correct.

It should be noted here that the term in question, given by (7), is an approximation to an integral which cannot be solved in closed form, which may account for some of the difference between (3a) and (6a)'. We should also point out that Fante has a sign error on this term in his (23), which may be seen by examining (19) and (20) of his paper.
The conductance term which appears in Fante's paper has also been incorrectly obtained, as a result of approximating the Hankel function of order zero and small argument, by the Neumann function alone. This can be seen by noting that the conductance term Fante obtains comes from (1) from the integration range $\beta>k$, where, as has been previously mentioned, the conductance contribution is zero. The same result for the conductance as Fante obtains is found, however, from the integration range 0 to $k$, by using both the small argument Bessel and Neuman function expressions in $H_{(2)}$, so that Fante's result for the conductance, which is

$$
\bar{G}=-\sqrt{\frac{\epsilon}{\mu}} \frac{\pi}{\ln (\Gamma K / \sqrt{2})}
$$

is correct.

The value of $\bar{G}$ obtained from (8), the approximate result of Fante, is at $1 \mathrm{MHz}$ for the antenna being considered, $\bar{G}=1.01813 \times 10^{-3}$ mhos, which is quite close to the calculated value obtained without approximation from (1), given in table 1 .

Further results obtained from integrating (1) are given in figure 2 and table 2 for the admittance as a function of $K,(a$ is constant at $1 \mathrm{~cm})$, for $\delta=0.1 \mathrm{~cm}$, along with results of Duncan, denoted by the subscript $D$, and values obtained from (4)' for the susceptance and (8) for the conductance, which we denote by the subscript $F$, after Fante.

It may be seen that for small $K$, the conductance is at first growing more rapidly than the susceptance with increasing $K$, but that finally the susceptance begins to overtake the conductance, reflecting the increasing importance of the $\ln (k \delta)$ term in the susceptance. Note that our conductance results check those of Duncan, but that Duncan's susceptance values are slightly lower, as a result of the way in which Duncan defines the susceptance, which he obtains from the current for a gap of zero thickness. We see that the $\bar{G}_{F}$ values agree very well with ours for $K<0.04$, but begin to differ appreciably for larger $K$, which is not surprising, since Fante's derivation assumes $K \ll 1$. The $B_{F}$ values are about 40 percent larger than the $B$ values for the smallest $K$ used, and about 5 percent larger at the largest $K$, with the best agreement in the range $K=0.01$ to 0.04 .

In figure 3 and table 3 are presented susceptance values as a function of $\delta$ for $K=0.15$. Using these results, we derive the following values for $B_{0}$ and $\Delta B$ from (2),

$$
\begin{aligned}
B_{0} & =3.0874 \times 10^{-3} \text { mhos } \\
\Delta B & =-8.0646 \times 10^{-4} \text { mhos. }
\end{aligned}
$$

Corresponding values from the approximate expressions of $(5 a)^{\prime}$ and $(5 b)$ are

$$
\tilde{B}_{0}=3.4962 \times 10^{-3} \text { mhos }
$$




$$
\Delta \tilde{B}=-7.9632 \times 10^{-4} \text { mhos }
$$

which are in better agreement with the values obtained from our calculations than was the case for $K$ $=2.0958 \times 10^{-4}$. We see that the susceptance is more sensitive to $\delta$ for the larger value of $K$, as shown by the larger $\Delta B$ term in the latter case.

It is interesting to note that the antenna conductance is determined by $K$ alone, and that changes in radius or frequency are indistinguishable, but that the susceptance depends individually upon each parameter. This may be seen more clearly by rewriting $(4)^{\prime}$ and (8) as

$$
\begin{gathered}
\widetilde{B}=-\frac{2 K}{\eta}\left\{0.9[2 K \ln (\Gamma K / \sqrt{2})]^{-1}\right. \\
+\ln (K / 2)+\ln (\delta / a)\} \\
\bar{G}=-\frac{\pi}{\eta \ln (\Gamma K / \sqrt{2})}
\end{gathered}
$$

with $\eta=\sqrt{\mu / \epsilon}$. Thus, the determining parameter for the conductance is $K$, while for the susceptance, the $\ln (\delta / a)$ term, contains the additional dependence upon the ratio of gap width to cylinder radius.

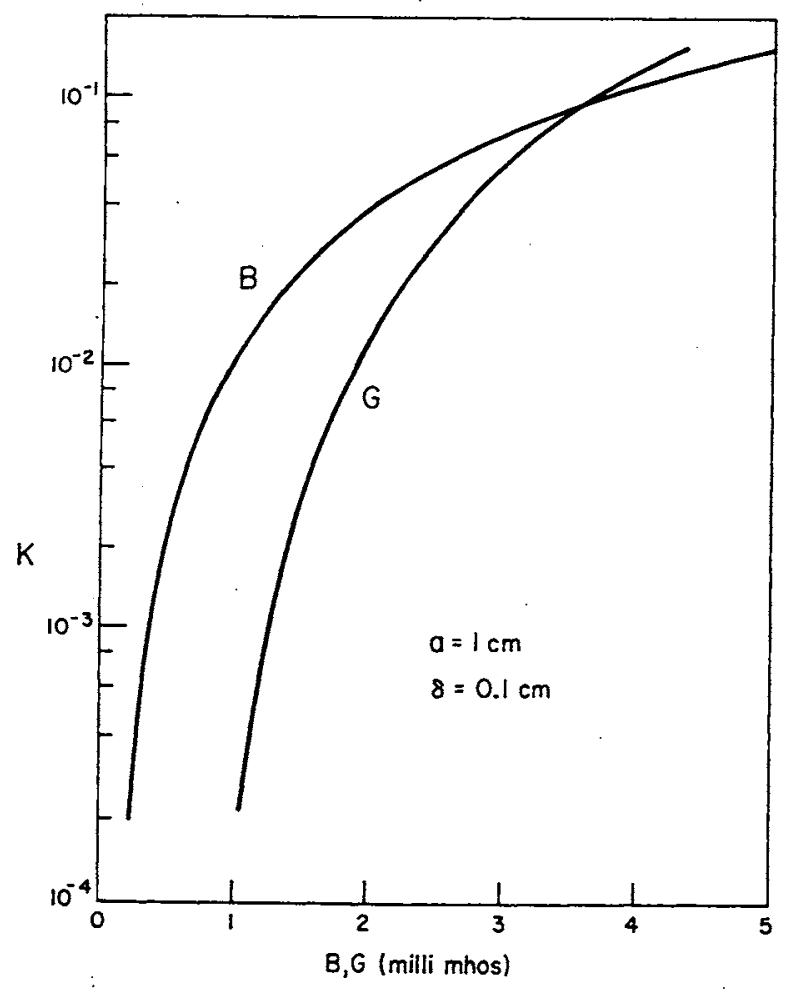

FICURE 2. The antenna admittance as a function of $\mathrm{K}$ with

\begin{tabular}{|c|c|c|c|c|c|c|}
\hline \multicolumn{7}{|c|}{$a=1 \mathrm{~cm} ; \delta=0.1 \mathrm{~cm}$} \\
\hline \multirow[b]{2}{*}{$k$} & \multicolumn{3}{|c|}{$\times 10^{3}$} & \multicolumn{3}{|c|}{$x 10^{\circ}$} \\
\hline & $G$ & $\bar{G}_{n}$ & $\hat{G}_{r}$ & $B$ & $\bar{B}_{i z}$ & $\vec{B}_{F}$ \\
\hline $2.0958 \times 10^{-4} \ldots \ldots \ldots$ & 1.0309 & & 1.0120 & 0.21870 & & 0.30269 \\
\hline $\begin{array}{l}4.1916 \times 10^{-4} \ldots \ldots \ldots \\
10^{-3} \ldots \ldots\end{array}$ & 1.1263 & …......... & 1.1050 & 0.26626 & .1....... & 0.34053 \\
\hline 001 & $\begin{array}{l}1.2720 \\
1.9204\end{array}$ & 1.92 & $\begin{array}{l}1.24099 \\
1.9063\end{array}$ & 0.95157 & 0.761 & $\begin{array}{l}0.94060 \\
0.94962\end{array}$ \\
\hline $0.02 \ldots$ & 2.2567 & 2.26 & 2.2652 & 1.3842 & 1.08 & 1.3824 \\
\hline $0.04 \ldots$ & 2.7220 & 2.72 & 2.7906 & 2.0852 & 1.55 & 2.1191 \\
\hline & 3.3973 & 3.40 & 3.6334 & 3.2466 & 2.43 & 3.3859 \\
\hline $0.15 \ldots$. & 4.3162 & 4.32 & 5.0040 & 4.9505 & 4.39 & 5.3298 \\
\hline
\end{tabular}
the radius a equal to $1 \mathrm{~cm}$ and an exciting gap thickness $\delta$ equal to $0.1 \mathrm{~cm}$.
TABLE 2

TABLE 3
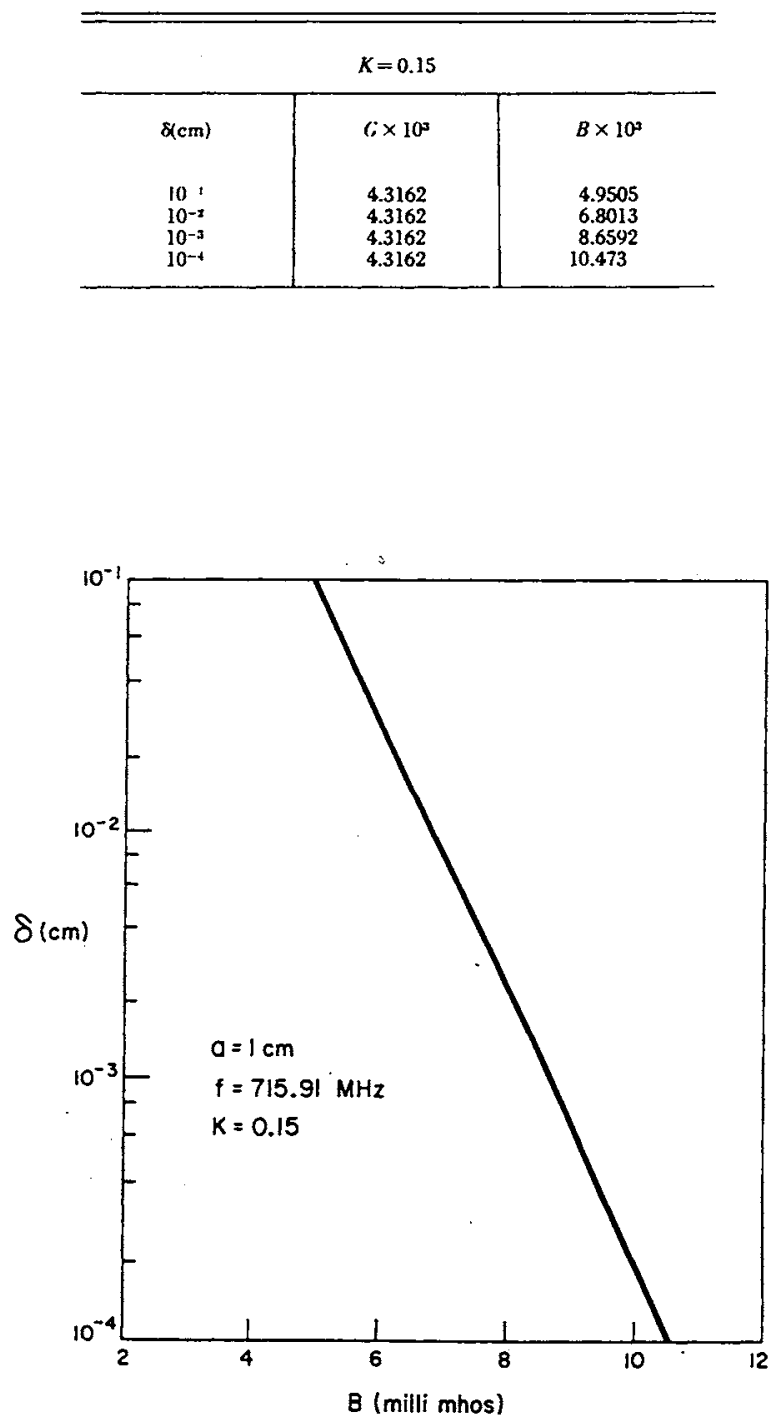

FIGURE 3. Variation of susceptance $\mathrm{B}$ as a function of exciting gap thickness $\delta$, at $715.7 \mathrm{MHz}(\mathrm{K}=0.15)$ with an antenna radius a of $1 \mathrm{~cm}$. 
The calculated results presented in figures 1 and 3 show that the sensitivity of the susceptance to changing gap width is greater for the larger value of $K$, a result which is shown by (1la). For large enough $K$ and/or small enough $\delta$, the first term in (1la) becomes small in comparison with the others, and $\widetilde{B}$ then is well approximated by (4), the result of Chen and Keller. It is interesting to note that if we divide (4) by $2 \pi a$, we obtain

$$
b=\frac{\tilde{B}}{2 \pi a}=-2 f \epsilon \ln (k \delta / 2),
$$

which is approximately the susceptance per unit length for a slot in an infinite plane. Thus, it is not surprising to find that (4) becomes more accurate as $K$ increases while at the same time being inadequate for the smaller $K$ values, where the correction term (7) is important.

\section{Conclusion}

A straightforward numerical technique has been described which allows the evaluation without approximation of the admittance of an infinite cylindrical antenna as a function of the exciting gap thickness. The results obtained are in agreement with those of
Duncan for the conductance, but have for the susceptance the additional advantage of explicitly showing the dependence of the susceptance upon the feeding gap thickness, which Duncan's results do not show. It has been shown, in addition, that the approximate form derived by Chen and Keller, and Fante, for the antenna susceptance, neglects a term in the susceptance which does not contain the gap thickness. Good agreement is obtained between the numerical results presented here and an approximate form for the susceptance, which includes this neglected term. The approximate form for the conductance derived by Fante has been found to give results which agree well with the calculated values obtained here.

\section{References}

Chen, Y. M., and J. B. Keller (1962), Current and input impedance of a cylindrical antenna, J. Res. NBS 66D (Radio Prop.) No. 1, $15-21$.

Duncan, R. H. (1962), Theory of the infinite cylindrical antenna including the feedpoint singularity in antenna current. J. Res. NBS 66D (Radio Prop.) No. 2, 181-187.

Fante, R. L. (1966a), On the admittance of the infinite cylindrical antenna, Radio Sci. 1 (New Series), Nu. 9. 1041-1044.

Fante, R. L. (1966b), Errata to above reference, Radio Sci., I (New Series), No. 10, 1234.

Ralston, A. (1965), A First Course in Numerical Analysis (McCrawHill Bouk Co., Inc., New Yurk, N.Y.).

(Paper 2-12-309) 